\title{
1 Novel Structural Features of Human Norovirus Capsid
}

2

3 Jessica Devant ${ }^{1,2}$, Götz Hofhaus ${ }^{3}$, and Grant S. Hansman ${ }^{1,2, \#}$

4

$5 \quad{ }^{1}$ Schaller Research Group at the University of Heidelberg and the DKFZ, Heidelberg,

6 Germany

$7 \quad{ }^{2}$ Department of Infectious Diseases, Virology, University of Heidelberg, Heidelberg,

8 Germany

$9 \quad{ }^{3}$ Bioquant, CellNetWorks, University of Heidelberg, Heidelberg, Germany

10

11 SHORT TITLE: Cryo-EM structure of GII.4 norovirus VLPs

12

13 "Corresponding author

14 Grant S. Hansman: CHS Foundation, University of Heidelberg, and German Cancer

15 Research Center, Heidelberg, Germany. Email: g.hansman@dkfz.de 
ABSTRACT

17 Human noroviruses are a major cause of gastroenteritis, yet there are still no vaccines

18 or antivirals available. Nevertheless, a number of vaccine candidates that are currently

19 in clinical trials are composed of norovirus virus-like particles (VLPs). These VLPs

20 are recognized as morphologically and antigenically similar to norovirus virions. An

21 X-ray crystal structure of the prototype (GI.1) VLPs showed that the norovirus capsid

22 has a $\mathrm{T}=3$ icosahedral symmetry and is composed of 180 copies of the major capsid

23 protein (VP1) that folds into three quasi-equivalent subunits (A, B, and C). In this

24 study, we determined the cryo-EM structure of VLPs for two GII.4 noroviruses that

25 were detected in 1974 and 2012. We showed that these VLPs had a T=4 symmetry

26 and were composed of 240 copies of VP1. The VP1 on the T=4 VLPs adapted four

27 quasi-equivalent subunits (termed A, B, C, and D), which formed two distinct dimers

28 (A/B and C/D). We found that the $\mathrm{T}=4$ protruding domain was elevated $\sim 21 \AA$ off the

29 capsid shell, which was $\sim 7 \AA$ more than the previously determined for the T=3 GII.10

30 norovirus. Another interesting feature of the $T=4$ VLPs was a small cavity and flap-

31 like structure located at the twofold axis. This structural feature was associated with

32 the shell domain (D subunit) and disrupted the contiguous shell. Altogether, we

33 showed that the $\mathrm{T}=4$ VLPs had a number of structural similarities and differences

34 with other noroviruses, but how these structural changes associate with norovirus

35 virions could be important for vaccine studies. 
IMPORTANCE

37 The discovery that the GII.4 VLPs (identified in 1974 and 2012, termed CHDC-1974

38 and NSW-2012, respectively) have a T=4 symmetry is of major significance, since

39 the NSW-2012 is clinically important and previous structural and biochemical studies

40 assumed noroviruses have a $\mathrm{T}=3$ symmetry and are composed of 180 copies of VP1.

41 More importantly, NSW-2012 norovirus shared 96\% amino acid identity with a GII.4

42 vaccine candidate and our data suggests that this vaccine might also have a $\mathrm{T}=4$

43 symmetry. Although it is not clear if the $T=4$ VLPs were an artifact of the insect cell

44 expression system, the $\mathrm{T}=4 \mathrm{VLP}$ vaccines might not recognize equivalent epitopes on

$45 \mathrm{~T}=3$ virions, which will be important for future neutralization studies. Finally, further

46 studies with other norovirus genotypes and virions are clearly needed in order to determine the level of this structural diversity. 
INTRODUCTION

50 Human noroviruses are members of the Caliciviridae family and are a leading cause

51 of outbreaks of acute gastroenteritis. The virus has a positive sense, single stranded 52 RNA genome of $\sim 7.7 \mathrm{kbp}$. The genome is organized into three open reading frames 53 (ORFs), where ORF1 encodes nonstructural proteins and ORF2 and ORF3 encode a 54 major structural protein (termed VP1) and a minor structural protein (termed VP2), respectively. Noroviruses are genetically diverse and based on VP1 sequences there are seven genogroups (GI-GVII), where GI, GII, and GIV cause infections in humans $(1,2)$. The GI and GII are further subdivided into numerous genotypes, with GII genotype 4 (GII.4) recognized as the most prevalent and clinically important $(3,4)$.

59

60 Development of an effective norovirus vaccine or antiviral has been hampered by the

61 lack of a suitable cell culture system or small animal model. Moreover, the extensive 62 genetic and antigenic diversity likely hinders vaccine development. Nevertheless, 63 several vaccine candidates have progressed to phase I and II human clinical trials. 64 Most vaccines were composed of norovirus GII.4 or GI.1 virus-like particles (VLPs), 65 which can be produced by expressing VP1 in insect cells. These vaccines were well 66 tolerated, highly immunogenic, and appeared to be safe, since they did not comprise 67 of live or attenuated virus. However, limitations of the current vaccine formulations 68 included mild norovirus like-symptoms, restricted long-term immunity, and limited cross-protection $(5,6)$.

71 Structural analysis of GI.1 VLPs reveals that VP1 is separated into two distinct 72 domains: a shell domain (S domain) that encloses the RNA and a protruding domain 
73 (P domain) that binds to co-factors, such as histo-blood group antigens (HGBAs) and

74 bile acids (7-9). A hinge region, which is typically composed of 10-14 amino acids,

75 also connects the $\mathrm{S}$ and $\mathrm{P}$ domains. The $\mathrm{P}$ domain has a $\beta$-barrel fold that is 76 structurally conserved in the Caliciviridae family. Dimerization of the $\mathrm{P}$ domains 77 forms arch shaped protrusions that can be seen using electron microscopy. The P 78 domain is further subdivided into $\mathrm{P} 1$ and $\mathrm{P} 2$ subdomain, where $\mathrm{P} 2$ subdomain appears 79 to be an insertion in the P1 subdomain and is the most variable region on the capsid (7). Structural studies have shown that caliciviruses have a common overall organization of $\mathrm{T}=3$ icosahedral symmetry and are comprised of 180 copies of VP1 (7, 10-13). On the virus particles, the VP1 forms three quasi-equivalent subunits, termed A, B, and C (7). The norovirus $\mathrm{A}$ and $\mathrm{B}$ subunits assemble into 60 dimers (termed $\mathrm{A} / \mathrm{B}$ ) at the quasi twofold axis, whereas the $\mathrm{C}$ subunit assembles into $30 \mathrm{C} / \mathrm{C}$ dimers that are located at the strict icosahedral twofold axis. For the GI.1 VLPs, the A/B dimers have 88 a bent $\mathrm{S}$ domain conformation, whereas the $\mathrm{C} / \mathrm{C}$ dimers have flat $\mathrm{S}$ domain conformation (7). The conformational differences within these dimers likely

90 facilitates the curvature of the virus particle to form a closed shell, which is 91 commonly seen in other $\mathrm{T}=3$ icosahedral viruses (14). Interestingly, smaller norovirus 92 VLPs ( $25 \mathrm{~nm}$ in diameter) that are assumed to have a $\mathrm{T}=1$ icosahedral symmetry 93 were also reported $(15,16)$; however the structure of these smaller VLPs has not yet 94 been determined.

96 In this study, we determined the cryo-EM structure of VLPs for GII.4 variants that 97 were identified in 1974 and 2012, termed CHDC-1974 and NSW-2012, respectively 
98 (17, 18). We showed that these VLPs had a $\mathrm{T}=4$ icosahedral symmetry and were

99 composed of 240 copies of VP1. In order to form the T=4 symmetry, VP1 adapted

100 four quasi-equivalent subunits, termed $\mathrm{A}, \mathrm{B}, \mathrm{C}$, and $\mathrm{D}$, which subsequently gave rise

101 to two distinct dimers, termed A/B and C/D. These VLPs consisted of $60 \mathrm{~A} / \mathrm{B}$ dimers

102 and $60 \mathrm{C} / \mathrm{D}$ dimers, where at the icosahedral 2-fold axis, B, C, and D subunits were

103 alternating, while the A subunit was located at the fivefold axis. Altogether, our

104 findings showed that the GII.4 VLPs had structural modifications that might have

105 important implications for vaccine design. 
MATERIALS AND METHODS

107

\section{VLP preparation}

109 The NSW-2012 and CHDC-1974 VLPs (Genebank accession numbers JX459908 and

110 ACT76142, respectively) were expressed in a baculovirus system as previously

111 described (19-21). Briefly, the bacmid containing the recombinant VP1 gene was 112 transfected in Sf9 insect cells. After incubation for five days, the culture medium was 113 centrifuged for $10 \mathrm{~min}$ at $3,000 \mathrm{rpm}$ at $4^{\circ} \mathrm{C}$. The recovered baculovirus was 114 subsequently used to infect Hi5 insect cells. After five days post infection, the culture 115 medium was centrifuged for $10 \mathrm{~min}$ at $3,000 \mathrm{rpm}$ at $4{ }^{\circ} \mathrm{C}$ and then $1 \mathrm{~h}$ at $6,500 \mathrm{rpm}$ at $1164{ }^{\circ} \mathrm{C}$. The VLPs in the supernatant were concentrated by ultracentrifugation at 35,000 $117 \mathrm{rpm}$ for $2 \mathrm{~h}$ at $4{ }^{\circ} \mathrm{C}$ and then further purified using $\mathrm{CsCl}$ equilibrium gradient 118 ultracentrifugation at $35,000 \mathrm{rpm}$ for $18 \mathrm{~h}$ at $4^{\circ} \mathrm{C}$. To remove the $\mathrm{CsCl}$, the VLPs were 119 pelleted for $2 \mathrm{~h}$ at $40,000 \mathrm{rpm}$ at $4^{\circ} \mathrm{C}$ and subsequently resuspended in $\mathrm{PBS}$ (pH 7.4).

\section{Negative stain electron microscopy}

122 The integrity of the VLPs was confirmed by negative stain electron microscopy (EM).

123 GII.4 virions from stool were also examined using EM (prepared as above expect for 124 the $\mathrm{CsCl}$ equilibrium gradient ultracentrifugation step). Briefly, the VLPs were diluted 125 1:30 in distilled water and applied to EM grids, whereas the stool sample was diluted 126 to $10 \%$ with PBS, applied to EM grid, washed with water, and then fixed with $4 \%$ 127 glutaraldehyde. The grids were washed with distilled water, stained with $0.75 \%$ 128 uranyl acetate, and the excess uranyl acetate removed with filter paper. EM images 129 were acquired on a Zeiss 910 electron microscope at 50,000× magnification. 


\section{Cryo-EM data sample preparation and data collection}

132 UNSW-2012 and CHDC-1974 VLPs $(3 \mu \mathrm{l})$ were applied on freshly glow discharged

133 Quantifoil holey carbon support films (R1.2/1.3; Quantifoil) and blotted for 18

134 seconds at $100 \%$ humidity at $10^{\circ} \mathrm{C}$ before been plunged in liquid ethane using an FEI

135 Mark IV Vitrobot (Thermo Fischer Scientific). Vitrified specimens were imaged on a

136 Titan Krios operated at $300 \mathrm{keV}$. NSW-2012 micrographs were acquired with a K2

137 direct electron detector at $64,000 \times$ magnification, corresponding to a pixel size of

$1382.27 \AA / \mathrm{px}$, while CHDC-1974 micrographs were collected using a K3 camera with

139 Latitude $\mathrm{S}$ software (Gatan) at $64,000 \times$ magnification, corresponding to a pixel size of

$140 \quad 1.375 \AA / p x$.

Cryo EM data processing

143 Images were processed with Relion 2.1 software for NSW-2012 and cryosparc 144 software for CHDC-1974 (22, 23). Initially, the movies containing 16 frames for 145 NSW-2012 and 40 frames for CHDC-1974 were motion corrected using motioncor2 146 software (24) and contrast transfer function (CTF) was performed using ctffind 4.1 147 software (25). An initial set of 1,000 particles was manually picked for 2D 148 classification to produce averages suitable as references for automated particle 149 picking. The autopicked particles were sorted in a 2D classification step and the best 150 particles were used for calculation of an initial starting model, followed by 3D 151 classification. A subset of particles that generated the highest resolution was selected 152 for further refinement. The 3D refinement and post-processing of NSW-2012 from 15310,548 particles produced a final map at 7.3- $\AA$ resolution with I2 symmetry imposed 154 (0.143 FSC cutoff). For CHDC-1974, a subset of 42,485 particles for refinement 155 revealed a map of 6.1 - $\AA$ resolution using the 0.143 FSC cutoff. Cryo-EM VLP 
156 structures for CHDC-1974 (accession number: EMD-4549) and NSW-2012 (EMD-

157 4550) were deposited at EMDB.

158

159 Fitting of the X-ray structures into the density maps

160 Crystal structures of NSW-2012 P domain (PDB ID: 4OOS) and CHDC-1974 P

161 domain (5IYN) were fitted into the respective densities using the "fit in map"

162 command in the UCSF Chimera software (26). Since a high-resolution GII.4 shell

163 domain was unavailable, the GI.1 Norwalk virus S domain was extracted from the X-

164 ray crystal structure (1IHM) and fitted into the GII.4 cryo-EM density using UCSF

165 Chimera software. 
166

167

168

169

170

\section{RESULTS AND DISCUSSION}

The purpose of this study was to analyze the GII.4 VLP architecture of NSW-2012 and CHDC-1974 and then relate the findings with known GI.1 and GII.10 VLP structures. The NSW-2012 VP1 sequence had a single amino acid insertion at position 394 (NSW-2012 numbering) compared to CHDC-1974 (Fig. 1). Overall, NSW-2012 and CHDC-1974 shared 89\% amino acid identity. Most (45 of 54) amino acid substitutions were located in the P domain. Negative stain EM images revealed that these the VLPs exhibited characteristic norovirus morphology (Fig. 2). However, the diameter of these VLP images was measured to be $\sim 52 \mathrm{~nm}$, which suggested that the GII.4 VLPs were larger than GII.10 and GI.1 VLPs that had diameters of $\sim 43 \mathrm{~nm}$ and $38 \mathrm{~nm}$, respectively.

\section{Cryo-EM structure of GII.4 NSW-2012 VLPs}

The structure of the NSW-2012 VLPs was determined using cryo-EM. The VLPs were mono-dispersed in vitreous ice and appeared homogenous in size (Fig. 3A). From the 364 images, 10,548 particles were used for structural reconstruction and refined to $7.3 \AA$ resolution (Fig. 3B).

Unexpectedly, NSW-2012 VLPs were discovered to have a T=4 icosahedral symmetry (Fig. 4). Our data revealed that these VLPs were composed of 240 copies of VP1, rather than the 180 VP1 copies in GI.1 and GII.10 VLPs. The inner diameter of NSW-2012 shell was measured as $32 \mathrm{~nm}$, while the outer capsid diameter was 50 $\mathrm{nm}$. Interestingly, this large diameter of the $\mathrm{T}=4$ VLPs corresponded to an inner shell 
volume of $17,157 \mathrm{~nm}^{3}$, which was $\sim 2.6$ times the volume of the GII.10 VLPs that had an inner diameter of $23 \mathrm{~nm}$ (13).

Another interesting structural feature of these NSW-2012 VLPs was a small cavity and flap-like structure on the contiguous shell (Fig. 5). This feature was associated with the S domain and found on opposing sides at the twofold axis. Interestingly, NSW-2012 VLPs were capable of binding HBGAs and norovirus-specific antibodies (27, 28), which indicated that despite the cavity and flap-like structure these VLPs were biologically functional.

Structural analysis of the T=4 VLPs indicated that VP1 adapted four quasi-equivalent subunits, termed A, B, C, and D. Subsequently, these four subunits gave rise to two distinct dimers, termed A/B and C/D (Figs. 4 and 6). At the icosahedral twofold axis, $\mathrm{B}, \mathrm{C}$, and $\mathrm{D}$ subunits were alternating, while the A subunit was positioned at the fivefold axis (Fig. 4). Of major importance, the $\mathrm{T}=4$ VLPs comprised of $60 \mathrm{~A} / \mathrm{B}$ dimers and $60 \mathrm{C} / \mathrm{D}$ dimers, which was distinct from the T=3 GI.1 and GII.10 VLPs that assembled with $60 \mathrm{~A} / \mathrm{B}$ and $30 \mathrm{C} / \mathrm{C}$ dimers. We also observed that the $\mathrm{T}=4 \mathrm{~A} / \mathrm{B}$ and C/D dimers had a bent conformation at the bottom of the $\mathrm{S}$ domain, which was in contrast to the GI.1 VLPs that consisted of both bent (A/B) and flat $(\mathrm{C} / \mathrm{C})$ dimers (7). Likely, the bent $\mathrm{A} / \mathrm{B}$ and $\mathrm{C} / \mathrm{D}$ dimers facilitated the necessary curvature to form particles with a $\mathrm{T}=4$ symmetry.

In order to better comprehend how VP1 assembled into the T=4 VLPs, the X-ray crystal structure of NSW-2012 P domain (4OOS) and GI.1 S domain (1IHM) were fitted into the VLP density map. We found that the NSW-2012 P domain dimer could 
be unambiguously positioned into the VLP structure with cross correlation coefficient

217 not undergone any major structural modifications. In the case of the $\mathrm{S}$ domain, the

218 GI.1 S domain needed to be manually positioned into the density map. The GI.1 S

219 domain fitted well into to $\mathrm{A} / \mathrm{B}$ dimer and the $\mathrm{C}$ subunit, while the $\mathrm{D}$ subunit needed to

220 be further repositioned (Fig. 7B). This additional fitting in the D subunit was

221 necessary in order to occupy the elevated density of the cavity and flap-like regions.

Unfortunately, it was problematic to fit the hinge region, since the hinge region on the

224 X-ray crystal structure of NSW-2012 P domain was excluded from the expression

225 construct and the hinge region on the GI.1 VLPs was flattened (7, 13). Interestingly,

226 an additional density was also observed between the $\mathrm{S}$ domain and the C-terminus of

227 the P domain on the D subunit (Fig. 8). This connection appeared to stabilize the bent

228 conformation of the $\mathrm{C} / \mathrm{D}$ dimer and subsequently the $\mathrm{T}=4$ VLPs. Indeed, the $\mathrm{C}$ -

229 terminus of VP1 on the GI.1 VLPs and the GII.4 P domain were found to be flexible

230 (7, 28). Interestingly, the C-terminus of VP1 was previously shown to be important

231 for the size and stability of VLPs (29). Therefore, it is possible that GII.4 VP1

232 sequence has a remarkable ability to form $\mathrm{T}=4$ VLPs, which could provide an

233 additional advantage for this genotype.

235 Another interesting feature that we observed with the $\mathrm{T}=4$ VLPs was the raised $\mathrm{P}$ 236 domains (Figs. 4 and 8). We found that the T=4 P domain was elevated $\sim 21 \AA$ off the 237 shell, which was higher than the P domains on the GII.10 VLPs, which were raised $238 \sim 14 \AA$ (13). The hinge region in NSW-2012 and GII.10 (13) were both $\sim 10$ amino 239 acids and mainly conserved (30). This result suggested that the raised $\mathrm{P}$ domains 
might be a structural feature of GII noroviruses, since the $\mathrm{P}$ domains on the GI.1

241 VLPs were essentially resting on the shell (7).

\section{Cryo-EM structure of GII.4 CHDC-1974 VLPs}

244 Following these results, we proceeded to determine the cryo-EM structure of the 245 CHDC-1974 VLPs. The VLPs were mostly mono-dispersed and homogenous in size 246 (Fig. 9A). From 591 images, 42,485 particles were used for the structural 247 reconstruction that led to a final resolution of $6.1 \AA$ (Fig. 9B). Remarkably, the 248 CHDC-1974 VLPs also had a T=4 symmetry (Fig. 10). In fact, the CHDC-1974 VLP 249 structure closely resembled the structural features of NSW-2012 VLPs.

251 We found that CHDC-1974 VLPs were composed of 240 copies of VP1 that formed

252 the equivalent subunits $(\mathrm{A}, \mathrm{B}, \mathrm{C}$, and $\mathrm{D})$ and $\mathrm{A} / \mathrm{B}$ and $\mathrm{C} / \mathrm{D}$ dimers. The inner diameter 253 of the shell was $32 \mathrm{~nm}$, whereas the outer diameter of the capsid was $50 \mathrm{~nm}$. The 254 comparable small cavity and flap-like structures were also present on the CHDC-1974 255 VLPs (Fig. 11). The CHDC-1974 A/B and C/D dimers showed a similar bend as 256 NSW-2012 dimers, although slightly less pronounced (Fig. 12). The X-ray crystal 257 structure of CHDC-1974 P domain (5IYN) was easily fitted into the CHDC-1974 258 VLP density map (Fig. 13A). The GI.1 S domain also fitted into the A, B, and C 259 subunits, whereas the GI.1 S domain needed to be again repositioned to occupy the D 260 subunit (Fig. 13B). Similar to NSW-2012 VLPs, an additional density was observed 261 between the $\mathrm{S}$ and $\mathrm{P}$ domains on the D subunit (Fig. 14). Lastly, we found that 262 CHDC-1974 P domain was also lifted off the shell by 21 Å (Figs. 10 and 14). 
264 Overall, these results showed that GII.4 VP1 sequences isolated over three decades

265 apart remained structurally conserved. This could imply that other GII.4 VP1

266 sequences also form $\mathrm{T}=4$ VLPs when expressed in insect cells, especially since these

267 two sequences had only $89 \%$ amino acid identity. More importantly, the GII.4

268 noroviruses have dominated epidemics over the past decade and this structural feature

269 could represent a selective advantage over other GII genotypes that have a $\mathrm{T}=3$

270 capsid.

272 Structural implications of the $T=4$ VLPs

273 Our new discovery of these $\mathrm{T}=4$ GII.4 VLPs could have major implications for

274 vaccine development. Negative stain EM images of the GII.4c VLPs that are currently

275 tested in clinical trials showed the typical norovirus morphology $(6,31)$. However, the

276 size determination and the structure are not available.

278 In general, studies have shown that norovirus-specific antibody titers were raised after

279 vaccination with VLPs, but the levels of protection were not strongly improved 280 compared to placebo groups (32). It is tempting to speculate that the GII.4c VLPs 281 might also form $\mathrm{T}=4$ particles, since the GII.4c and NSW-2012 shared 94\% amino 282 acid identity; most (28 of 31 ) of the substitutions were located in the P domain; and 283 the hinge region was identical (Fig. 1). Therefore, this might suggest that the host 284 could produces neutralizing antibodies against epitopes on the $\mathrm{T}=4$ VLPs that were 285 not as accessible on $\mathrm{T}=3$ virions and that efficacy is difficult. Indeed, we have 286 identified several Nanobodies that bind to occluded regions on the T=4 GII.4 VLPs $287(30,33)$. 
289 In order to validate if the GII.4 virions actually assemble into $\mathrm{T}=3$ a cryo-EM

290 structure would be valuable, however norovirus virions are challenging to prepare in

291 large quantities. Nevertheless, when we compare EM images of GII.4 virions with

292 these $\mathrm{T}=4$ VLPs we found that the virions were smaller (Fig. 15). Therefore, our

293 preliminary results suggest that the GII.4 VLPs and virions were different in size and

294 likely other structural characteristics.

\section{Summary}

297 At least two important outcomes from this new discovery of these GII.4 T=4 VLPs

298 will be acknowledged. Firstly, the GII.4 VLPs might influence results from previous

299 studies that assumed norovirus VLPs were morphologically similar to virions. For 300 example, the molecular weight of the $\mathrm{T}=3$ and $\mathrm{T}=4$ VLPs would be $\sim 10.5 \mathrm{mDa}(180 \times$

301 VP1) and $\sim 14 \mathrm{mDa}(240 \times \mathrm{VP1})$, respectively. This mass difference would affect

302 results in mass spectrometry, Biacore, and isothermal titration calorimetry. Secondly,

303 the VLP vaccines that could be composed of $\mathrm{T}=4$ particles might produce

304 complicating immune responses. For example, the cavity and flap-like structures on

305 the $\mathrm{T}=4$ could elicit some antibodies that are not recognized by $\mathrm{T}=3$ virions.

306 Ultimately, when a patient is immunized with $\mathrm{T}=4$ VLPs, the immune response with

307 virions could effectively be lower. 
310 We acknowledge the excellence cluster CellNetworks (Cryo-EM network) of the

311 University of Heidelberg for cryo-EM data collection, the EM core facility at DKFZ,

312 and Baden-Württemberg High Performance Cluster (bwHPC). We thank David

313 Bhella for assistance with structural refinements; Anna Koromyslova for EM images

314 of GII.4 virions; and Benedikt Wimmer for setting up the cryo-EM software. The

315 funding for this study was provided by the CHS foundation; the Baden-Württemberg

316 Stiftung (GLYCAN-BASED ANTIVIRAL AGENTS); Deutsche

317 Forschungsgemeinschaft (DFG, FOR2327); and the BMBF VIP+ (Federal Ministry of

318 Education and Research) (NATION, 03VP00912). 
FIGURE LEGENDS

Figure 1. Amino acid sequence alignment of GII.4 VP1. NSW-2012 (JX459908), CHDC-1974 (ACT76142), and GII.4c (31) VP1 amino acid sequences were aligned

323 using ClusterX. The S domain (orange), hinge region (green), P1 subdomain (light

324 blue), and P2 subdomain (navy) were labeled accordingly. Compared to CHDC-1974,

325 NSW-2012 and GII.4c VP1 had a single amino acid insertion (arrow) at position 394

326 (NSW-2012 numbering). The S domain and hinge region were mainly conserved, 327 whereas most amino acid substitutions were located in the P2 subdomain.

Figure 2. EM images and hydrodynamic diameters of NSW-2012 and CHDC-

1974 GII.4 VLPs. Negative stain EM images of the GII.4 VLPs show the

331 characteristic norovirus morphology (50,000× magnification). The GI.1 West Chester 332 (AY502016.1) and GII.10 Vietnam026 (AF504671) VLPs are shown as reference (27, 333 34). The bar represents $100 \mathrm{~nm}$.

Figure 3. NSW-2012 cryo-EM data processing. (A) A representative cryo-EM micrograph of NSW-2012 VLPs at 64,000× magnification. The scale bar represents $100 \mathrm{~nm}$. (B) Gold standard Fourier shell correlation (FSC) plot of the icosahedral reconstruction of NSW-2012 indicates a resolution of $7.3 \AA$.

Figure 4. Cryo-EM reconstruction structure of NSW-2012 VLPs. The left side

341 shows NSW-2012 VLPs have a T=4 icosahedral symmetry (symmetry axis labeled 2,

342 3, and 5). These VLPs were composed of 240 copies of VP1. The VP1 adapted four 343 quasiequivalent conformations (A, B, C, and D) that gave rise to two distinct dimers 
344 (A/B and $\mathrm{C} / \mathrm{D}$ ). At the icosahedral twofold axis, the $\mathrm{B}, \mathrm{C}$, and $\mathrm{D}$ subunits were

345 alternating, while the A subunit is positioned at the fivefold axis. The right side shows

346 a cutaway section of these VLPs and indicates that the inner and outer diameters are

$34732 \mathrm{~nm}$ and $50 \mathrm{~nm}$, respectively. The P domains are elevated $\sim 21 \AA$ off the $\mathrm{S}$ domain.

Figure 5. NSW-2012 T=4 VLPs shows several new structural features. The cavity and flap-like structures are observed at the twofold axis and are found on opposing sides. The cavity and flap-like structures are associated with the $\mathrm{S}$ domain on the $\mathrm{D}$ 352 subunit.

Figure 6. NSW-2012 T=4 VLPs are formed with 60 A/B and 60 C/D VP1 dimers. The $\mathrm{A} / \mathrm{B}$ and $\mathrm{C} / \mathrm{D}$ dimers show an equivalent bent confirmation on the bottom of the $\mathrm{S}$ domain. An additional connection was observed between the $\mathrm{D}$ subunit of the $\mathrm{S}$ and $\mathrm{P}$ domain.

Figure 7. The X-ray crystal structures of NSW-2012 P domain and GI.1 S domain were fitted into the VLP density map. (A) The X-ray crystal structure of NSW-2012 P domain (4OOS, cartoon) could be fitted into the A/B and C/D P domain dimers, indicating little conformational change. (B) The X-ray crystal structure of the GI.1 S domain (1IHM, cartoon) fitted into the A/B and C/D S domain dimers.

364 However, the cavity and flap-like structures on the D subunit suggests a large conformational change compared to the typical $\mathrm{T}=3$ particles.

367 Figure 8. A close-up view of NSW-2012 C/D dimer. The fitted X-ray crystal 368 structures of the GI.1 S domain (cartoon) and the GII.4 P domain (cartoon) into the 

on the $\mathrm{D}$ subunit. (red).

cryo-EM map shows the how the hinge region connects the $\mathrm{S}$ and $\mathrm{P}$ domains. Also, the new connection between the $\mathrm{S}$ domain and the $\mathrm{C}$-terminus of the $\mathrm{P}$ domain is shown. The asterisk represents the missing hinge region on the X-ray crystal structures that connects of the $\mathrm{S}$ and $\mathrm{P}$ domains for the $\mathrm{C}$ subunit (blue) and D subunit

Figure 9. CHDC-1974 cryo-EM data processing. (A) A representative cryo-EM micrograph of CHDC-1974 VLPs at 64,000× magnification. The scale bar represents $100 \mathrm{~nm}$. (B) FSC plot of the icosahedral reconstruction of CHDC-1974 indicates a resolution of $6.1 \AA$ at 0.143 cutoff.

Figure 10. Cryo-EM structure and analysis of CHDC-1974 VLPs. The image on the left side shows that CHDC-1974 VLPs has a T=4 icosahedral symmetry and was composed of 240 copies of VP1. The VP1 adapted four quasiequivalent conformations (A, B, C, and D) that gave rise to two distinct dimers (A/B and C/D). At the icosahedral twofold axes, the B, C, and D subunits were alternating, while the A subunit was located around the fivefold axes. The right side shows a cutaway section of these VLPs and indicates that the inner and outer diameters are $32 \mathrm{~nm}$ and $50 \mathrm{~nm}$, respectively. The $\mathrm{P}$ domains are elevated $\sim 21 \AA$ off the $\mathrm{S}$ domain.

Figure 11. CHDC-1974 $\mathrm{T}=4$ VLPs shows several new structural features. The cavity and flap-like structures are observed at the twofold axis and are found on opposing sides. The cavity and flap-like structures are associated with the $\mathrm{S}$ domain 
Figure 12. CHDC-1974 T=4 VLPs are formed with $60 \mathrm{~A} / \mathrm{B}$ and $60 \mathrm{C} / \mathrm{D}$ VP1

dimers. The $\mathrm{A} / \mathrm{B}$ and $\mathrm{C} / \mathrm{D}$ dimers show an equivalent bent confirmation on the bottom of the $\mathrm{S}$ domain. An additional connection was observed between the $\mathrm{D}$ subunit of the $\mathrm{S}$ and $\mathrm{P}$ domain.

Figure 13. The X-ray crystal structures of CHDC-1974 P domain and GI.1 S domain were fitted into the VLP density map. (A) The X-ray crystal structure of NSW-2012 P domain (5IYN, cartoon) easily fitted into the A/B and C/D P domain dimers. (B) The X-ray crystal structure of the GI.1 S domain (1IHM, cartoon) fitted 403 into the $\mathrm{A} / \mathrm{B}$ and $\mathrm{C} / \mathrm{D} \mathrm{S}$ domain dimers. However, the cavity and flap-like structures 404 on the D subunit suggests a large conformational change from typical $\mathrm{T}=3$ particles.

Figure 14. A close-up view of CHDC-1974 C/D dimer. The fitted X-ray crystal 407 structures of the GI.1 S domain (cartoon) and the GII.4 P domain (cartoon) into the 408 cryo-EM map shows the how the hinge region connects the $\mathrm{S}$ and $\mathrm{P}$ domains. Also,

409 the new connection between the $\mathrm{S}$ domain and the $\mathrm{C}$-terminus of the $\mathrm{P}$ domain is 410 shown. The asterisk represents the missing hinge region on the X-ray crystal 411 structures that connects of the $\mathrm{S}$ and $\mathrm{P}$ domains for the $\mathrm{C}$ subunit (blue) and $\mathrm{D}$ subunit 412 (red).

414 Figure 15. EM images of GII.4 virions. Negative stain EM images of GII.4 virions 415 show that the virions exhibit a smaller diameter than GII.4 VLPs expressed in insect 416 cells. 
419 1. Pogan R, Schneider C, Reimer R, Hansman G, Uetrecht C. 2018. Norovirus-like VP1 particles exhibit isolate dependent stability profiles. Journal of physics. Condensed matter : an Institute of Physics journal 30:064006.

2. Hansman GS, Natori $K$, Shirato-Horikoshi H, Ogawa S, Oka T, Katayama K, Tanaka T, Miyoshi T, Sakae K, Kobayashi S, Shinohara M, Uchida K, Sakurai N, Shinozaki K, Okada M, Seto Y, Kamata K, Nagata N, Tanaka K, Miyamura T, Takeda N. 2006. Genetic and antigenic diversity among noroviruses. The Journal of general virology

3. Donaldson EF, Lindesmith LC, Lobue AD, Baric RS. 2008. Norovirus pathogenesis: mechanisms of persistence and immune evasion in human populations. Immunol Rev 225:190-211.

4. Choi SC, Simhadri VR, Tian L, Gil-Krzewska A, Krzewski K, Borrego F, Coligan JE. 2011. Cutting edge: mouse CD300f (CMRF-35-like molecule-1) recognizes outer membrane-exposed phosphatidylserine and can promote phagocytosis. Journal of immunology 187:3483-3487. 2011. Norovirus vaccine against experimental human Norwalk Virus illness. The New England journal of medicine 365:2178-2187. 
440 6. Clayton JS, Bolinger HK, Jaykus LA. 2018. Disinfectant testing against

441 human norovirus surrogates-What infection preventionists need to know.

442 Infection control and hospital epidemiology:1-2.

443 7. Prasad BV, Hardy ME, Dokland T, Bella J, Rossmann MG, Estes MK.

444 1999. X-ray crystallographic structure of the Norwalk virus capsid.

$445 \quad$ Science 286:287-290.

446 8. Kilic T, Koromyslova A, Hansman GS. 2019. Structural Basis for Human $447 \quad$ Norovirus Capsid Binding to Bile Acids. Journal of virology 93.

448 9. Tan M, Jiang X. 2005. The $\mathrm{p}$ domain of norovirus capsid protein forms a 449 subviral particle that binds to histo-blood group antigen receptors. $450 \quad$ Journal of virology 79:14017-14030.

451 10. Bhella D, Goodfellow IG. 2011. The cryo-electron microscopy structure of feline calicivirus bound to junctional adhesion molecule $\mathrm{A}$ at 9angstrom resolution reveals receptor-induced flexibility and two distinct conformational changes in the capsid protein VP1. Journal of virology

11. Chen R, Neill JD, Noel JS, Hutson AM, Glass RI, Estes MK, Prasad BV. 2004. Inter- and intragenus structural variations in caliciviruses and their functional implications. Journal of virology 78:6469-6479.

12. Kilic T, Koromyslova A, Malak V, Hansman GS. 2018. Atomic Structure of the Murine Norovirus Protruding Domain and Soluble CD300lf Receptor Complex. Journal of virology 92.

13. Hansman GS, Taylor DW, McLellan JS, Smith TJ, Georgiev I, Tame JR, 
noroviruses by a monoclonal antibody that binds to a site occluded in the viral particle. Journal of virology 86:3635-3646.

14. Melgaco FG, Correa AA, Ganime AC, Brandao MLL, Medeiros VM, Rosas CO, Lopes S, Miagostovich MP. 2018. Evaluation of skimmed milk flocculation method for virus recovery from tomatoes. Brazilian journal of microbiology : [publication of the Brazilian Society for Microbiology].

15. Ettayebi K, Crawford SE, Murakami K, Broughman JR, Karandikar U, Tenge VR, Neill FH, Blutt SE, Zeng XL, Qu L, Kou B, Opekun AR, Burrin human noroviruses in stem cell-derived human enteroids. Science 353:1387-1393.

16. Forest KT, Filutowicz MS. 2003. Remodeling of replication initiator proteins. Nature structural biology 10:496-498.

17. Eden JS, Tanaka MM, Boni MF, Rawlinson WD, White PA. 2013. Recombination within the pandemic norovirus GII.4 lineage. Journal of virology 87:6270-6282.

18. Bok K, Abente EJ, Realpe-Quintero M, Mitra T, Sosnovtsev SV, Kapikian AZ, Green KY. 2009. Evolutionary dynamics of GII.4 noroviruses over a 34-year period. Journal of virology 83:11890-11901.

19. Hansman GS, Natori K, Oka T, Ogawa S, Tanaka K, Nagata N, Ushijima recombinant capsid proteins. Archives of virology 150:21-36. 
21. Koromyslova AD, Hansman GS. 2017. Nanobodies targeting norovirus capsid reveal functional epitopes and potential mechanisms of neutralization. PLoS pathogens 13:e1006636.

493 22. Kabsch W. 2010. XDS. Acta Cryst. D66:125-132.

23. Sedji MI, Varbanov M, Meo M, Colin M, Mathieu L, Bertrand I. 2018. Quantification of human adenovirus and norovirus in river water in the north-east of France. Environmental science and pollution research international.

24. Pyrkov TV, Chugunov AO, Krylov NA, Nolde DE, Efremov RG. 2009. PLATINUM: a web tool for analysis of hydrophobic/hydrophilic organization of biomolecular complexes. Bioinformatics 25:1201-1202.

25. McCoy AJ G-KR, Adams PD, Winn MD, Storoni LC, Read RJ. . 2007. Phaser crystallographic software. Journal of Applied Crystallography. 40:658-674.

504 26. Emsley P LB, Scott WG, Cowtan K. 2010. Features and development of Coot. Acta Crystallographica Section D: Biological Crystallography. 66:486-501.

507 27. Koromyslova A, Tripathi S, Morozov V, Schroten H, Hansman GS. 2017. Human norovirus inhibition by a human milk oligosaccharide. Virology 508:81-89.

510 28. Singh BK, Leuthold MM, Hansman GS. 2015. Human noroviruses' fondness for histo-blood group antigens. Journal of virology 89:20242040. 
29. Bertolotti-Ciarlet A, White LJ, Chen R, Prasad BV, Estes MK. 2002. Structural requirements for the assembly of Norwalk virus-like particles. Journal of virology 76:4044-4055.

30. Koromyslova AD, Hansman GS. 2015. Nanobody binding to a conserved epitope promotes norovirus particle disassembly. Journal of virology 89:2718-2730.

31. Parra GI, Bok K, Taylor R, Haynes JR, Sosnovtsev SV, Richardson C, Green KY. 2012. Immunogenicity and specificity of norovirus Consensus GII.4 virus-like particles in monovalent and bivalent vaccine formulations. Vaccine 30:3580-3586.

32. Bernstein DI, Atmar RL, Lyon GM, Treanor JJ, Chen WH, Jiang X, Vinje J, Gregoricus N, Frenck RW, Jr., Moe CL, Al-Ibrahim MS, Barrett J, Ferreira J, Estes MK, Graham DY, Goodwin R, Borkowski A, Clemens R, Mendelman PM. 2015. Norovirus vaccine against experimental human GII.4 virus illness: a challenge study in healthy adults. The Journal of infectious diseases 211:870-878.

33. Koromyslova A, Hansman GS. 2017. Nanobodies targeting norovirus capsid reveal functional epitopes and potential mechanisms of neutralization. PLoS pathogens In press.

34. Hansman GS, Doan LT, Kguyen TA, Okitsu S, Katayama K, Ogawa S, Natori K, Takeda N, Kato Y, Nishio O, Noda M, Ushijima H. 2004. Detection of norovirus and sapovirus infection among children with gastroenteritis in Ho Chi Minh City, Vietnam. Archives of virology 149:1673-1688. 
bioRxiv preprint doi: https://doi.org/10.1101/528240; this version posted January 25, 2019. The copyright holder for this preprint (which was not certified by peer review) is the author/funder, who has granted bioRxiv a license to display the preprint in perpetuity. It is made available under aCC-BY-NC-ND 4.0 International license.

\section{8}


NSW-2012

CHDC-1974

GII.4C

NSW-2012

CHDC-1974

GII.4C

NSW-2012

CHDC-1974

GII.4C

NSW-2012

CHDC-1974

GII.4C

NSW-2012

CHDC-1974

GII.4C

NSW-2012

CHDC-1974

GII.4C

NSW-2012

CHDC-1974

GII.4C

NSW-2012

CHDC-1974

GII.4C

NSW-2012

CHDC-1974

GII.4C
1 MKMASSDANPSDGSAANLVPEVNNEVMALEPVVGAAIAAPVAGQQNVIDPWIRNNFVQAP

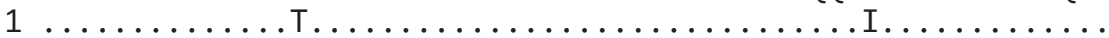

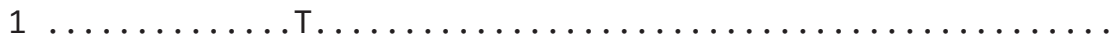

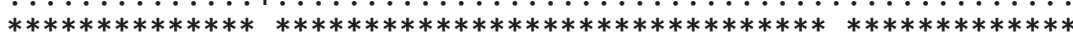

61 GGEFTVSPRNAPGEILWSAPLGPDLNPYLSHLARMYNGYAGGFEVQVILAGNAFTAGKVI

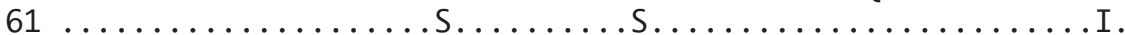

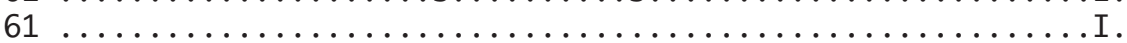

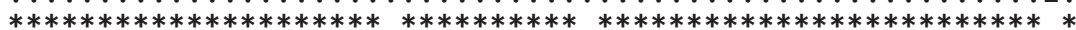

121 FAAVPPNFPTEGLSPSQVTMFPHIVVDVRQLEPVLIPLPDVRNNFYHYNQSNDPTIKLIA

$121 \ldots$........................................

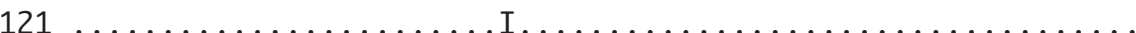

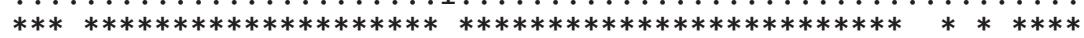

181 MLYTPLRANNAGDDVFTVSCRVLTRPSPDFDFIFLVPPTVESRTKPFSVPVLTVEEMTNS

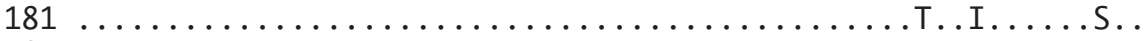

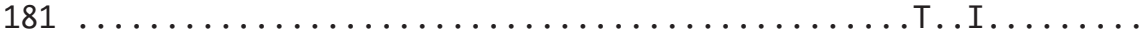

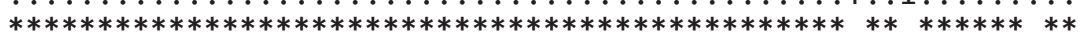

241 RFPIPLEKLFTGPSSAFVVQPQNGRCTTDGVLLGTTQLSPVNICTFRGDVTHITGSRNYT

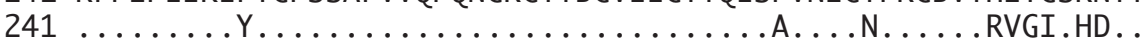

$241 \ldots \ldots \ldots \ldots \ldots$. . . . . . . . . . . . . . . . . . . TQE.

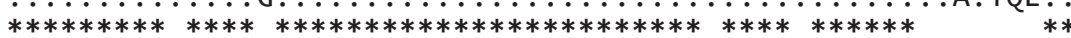

301 MNLASQNWNDYDPTEEIPAPLGTPDFVGKIQGVLTQTTRTDGSTRGHKATVYTGSADFAP

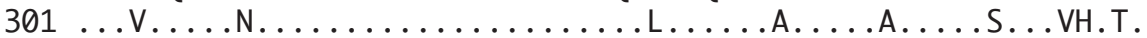

$301 \ldots . . \ldots$.................................. . VH.T. *** $* * * * * \quad * * * * * * * * * * * * * * * * * * * * * * \quad * * * * * * \quad * * * * * * * * * * * * * \quad * *$

361 KLGRVQFETDTDRDFEANQNTKFTPVGVIQDGGTTHRNEPQQWVLPSYSGRNTHNVHLAP

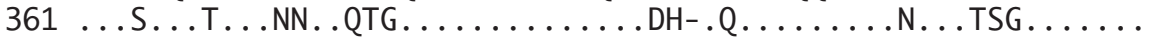

$361 \ldots$....S...SN ..TG ........V...S...Q........ ...DS ..... *** $* * * \quad * * * \quad * * \quad * * * * * * * * * * \quad * * * \quad \mathbf{4}^{*} * * * * * * * * * \quad * * * \quad * * * * * * *$ 60

60

60

120

120

120

180

180

180

240

240

240

300

300

300

360

360

360

420

419

420

421 AVAPTFPGEQLLFFRSTMPGCSGYPNMDLDCLLPQEWVQYFYQEAAPAQSDVALLRFVNP

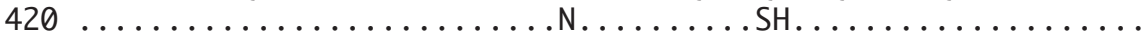

480

479

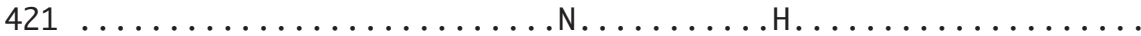

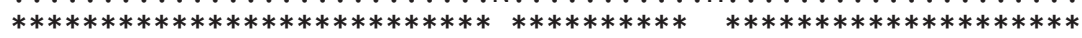

480

481 DTGRVLFECKLHKSGYVTVAHTGQHDLVIPPNGYFRFDSWVNQFYTLAPMGNGTGRRRAV

540

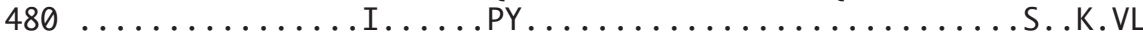

539

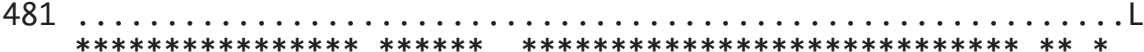

540 
Figure 2

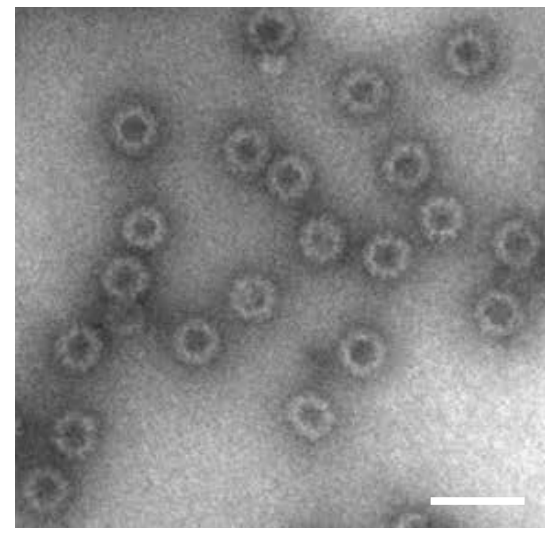

GII.4 NSW-2012

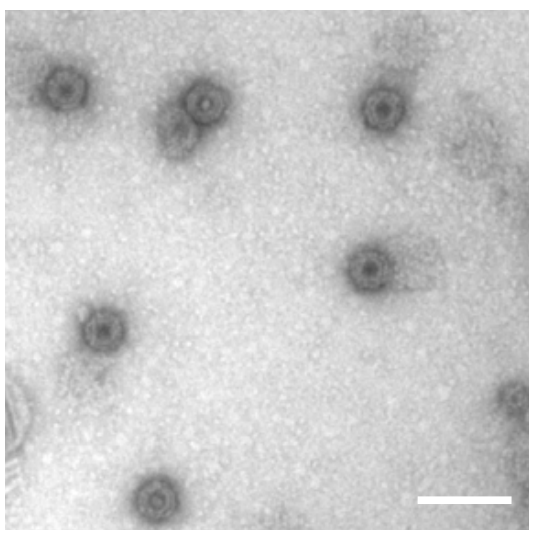

GI.1 West Chester

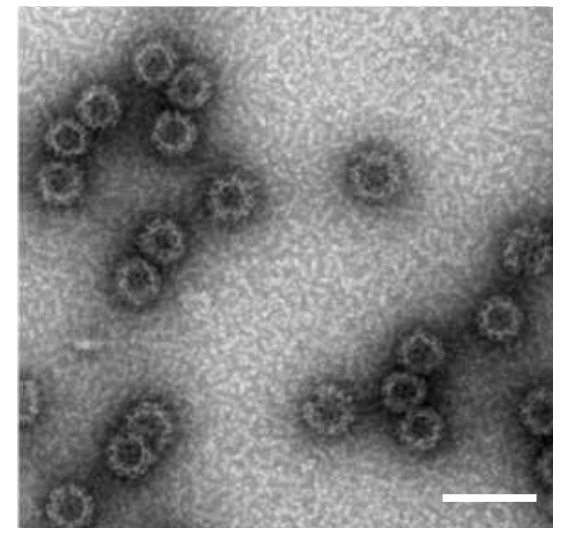

GII.4 CHDC-1974

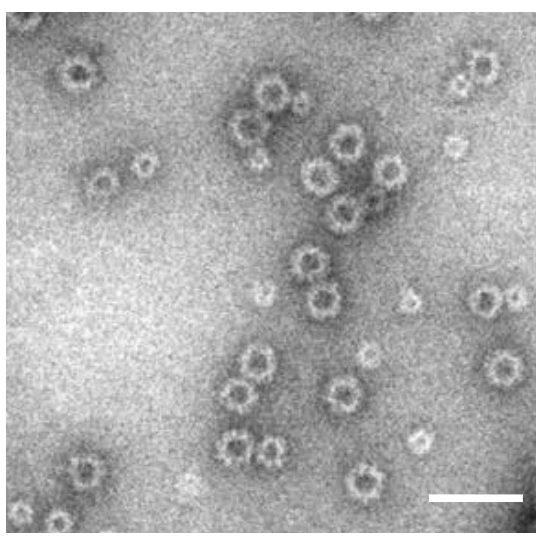

GII.10 026-Vietnam 
Figure 3

A

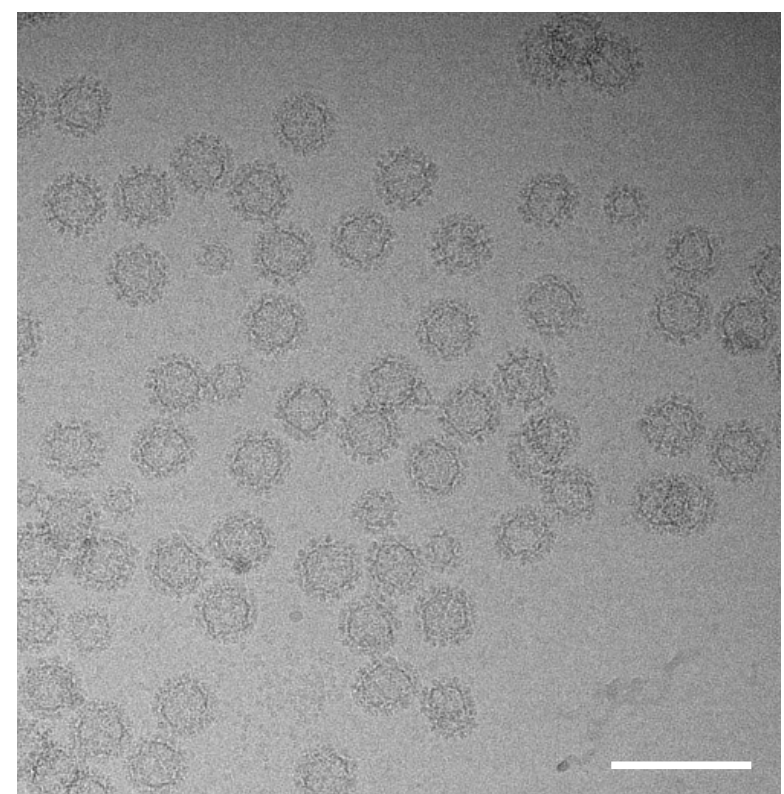

B

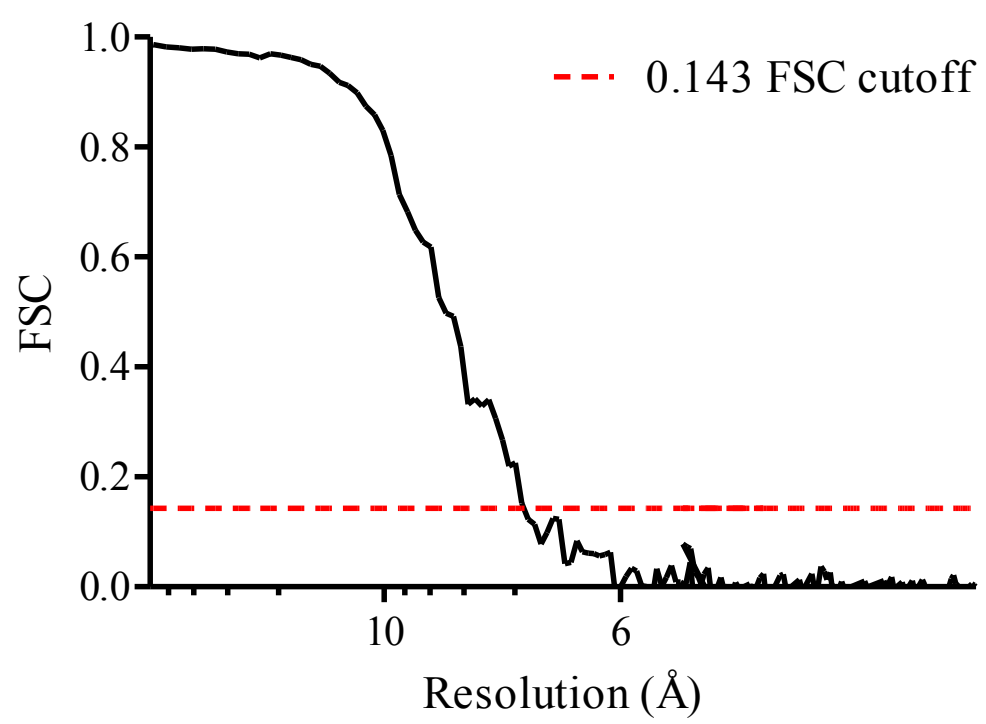


Figure 4
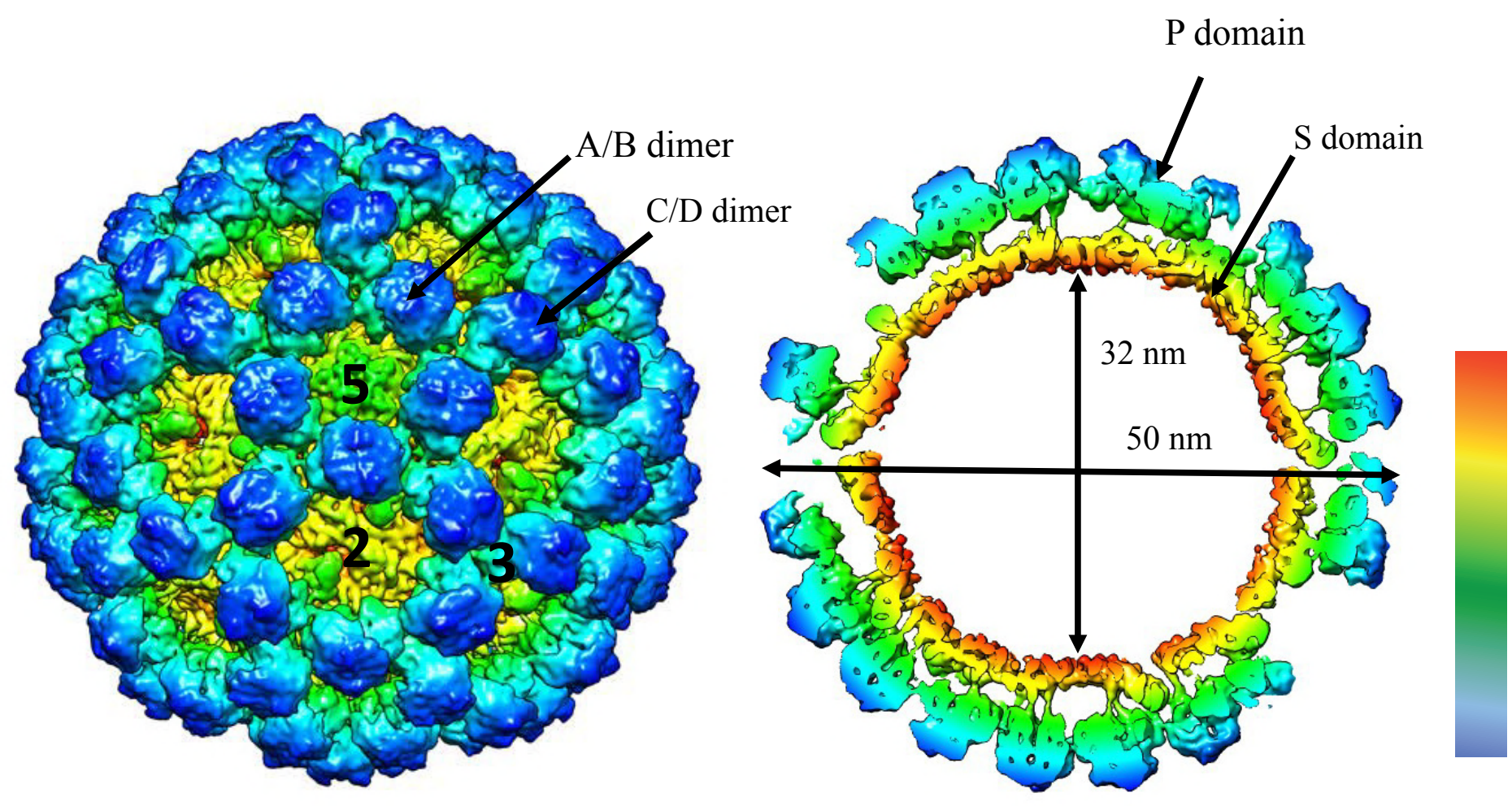

145-Å

175-尺̊

200-Å

225-Å

254- $\AA$ 
Figure 5

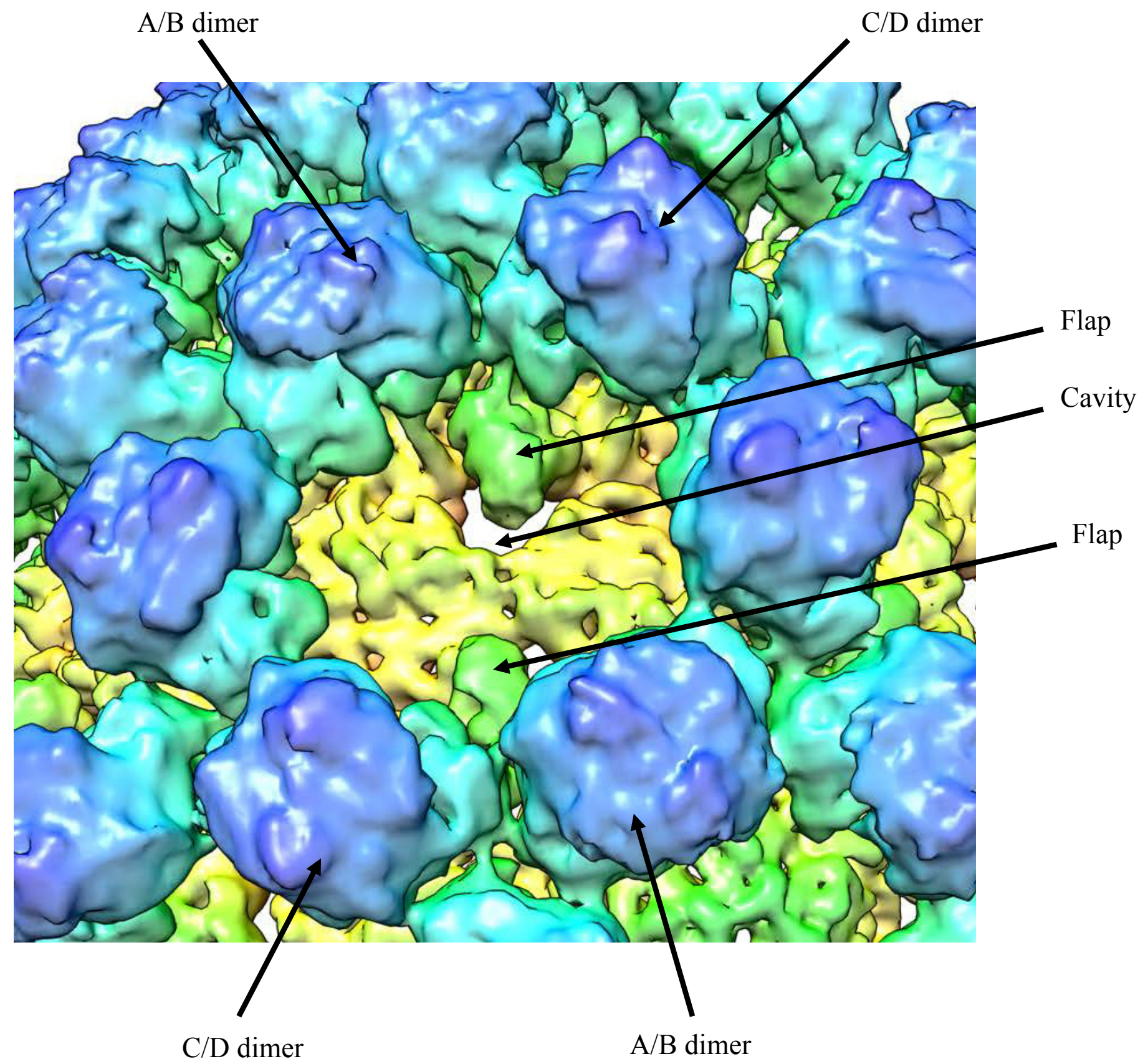


Figure 6

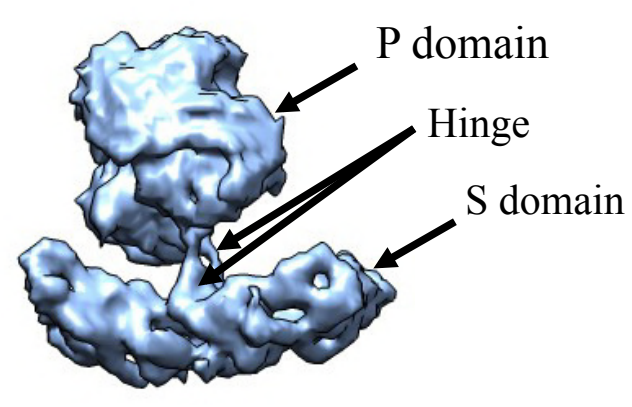

$\mathrm{A} / \mathrm{B}$ dimer

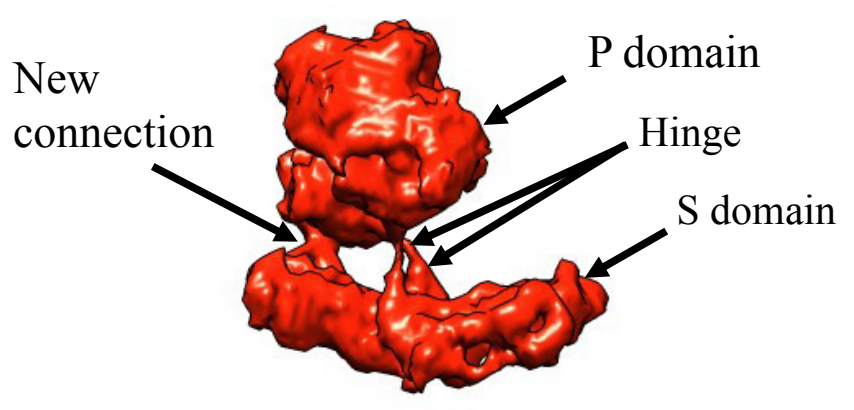

C/D dimer 
A
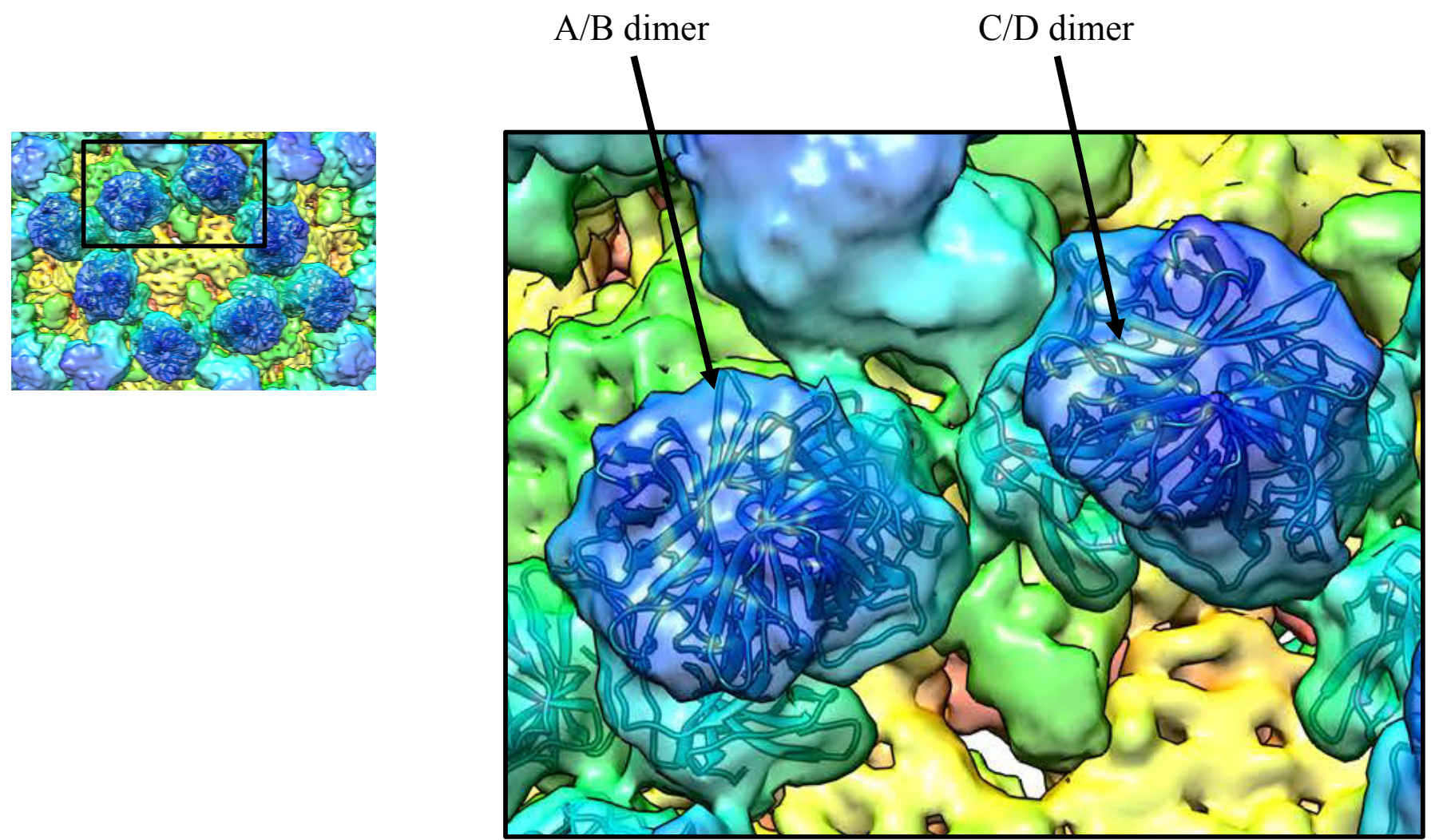

B

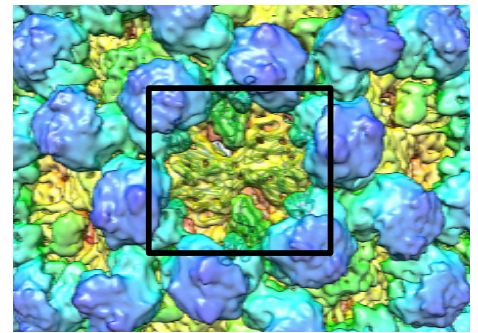

$\mathrm{A} / \mathrm{B}$ dimer $\quad \mathrm{C} / \mathrm{D}$ dimer

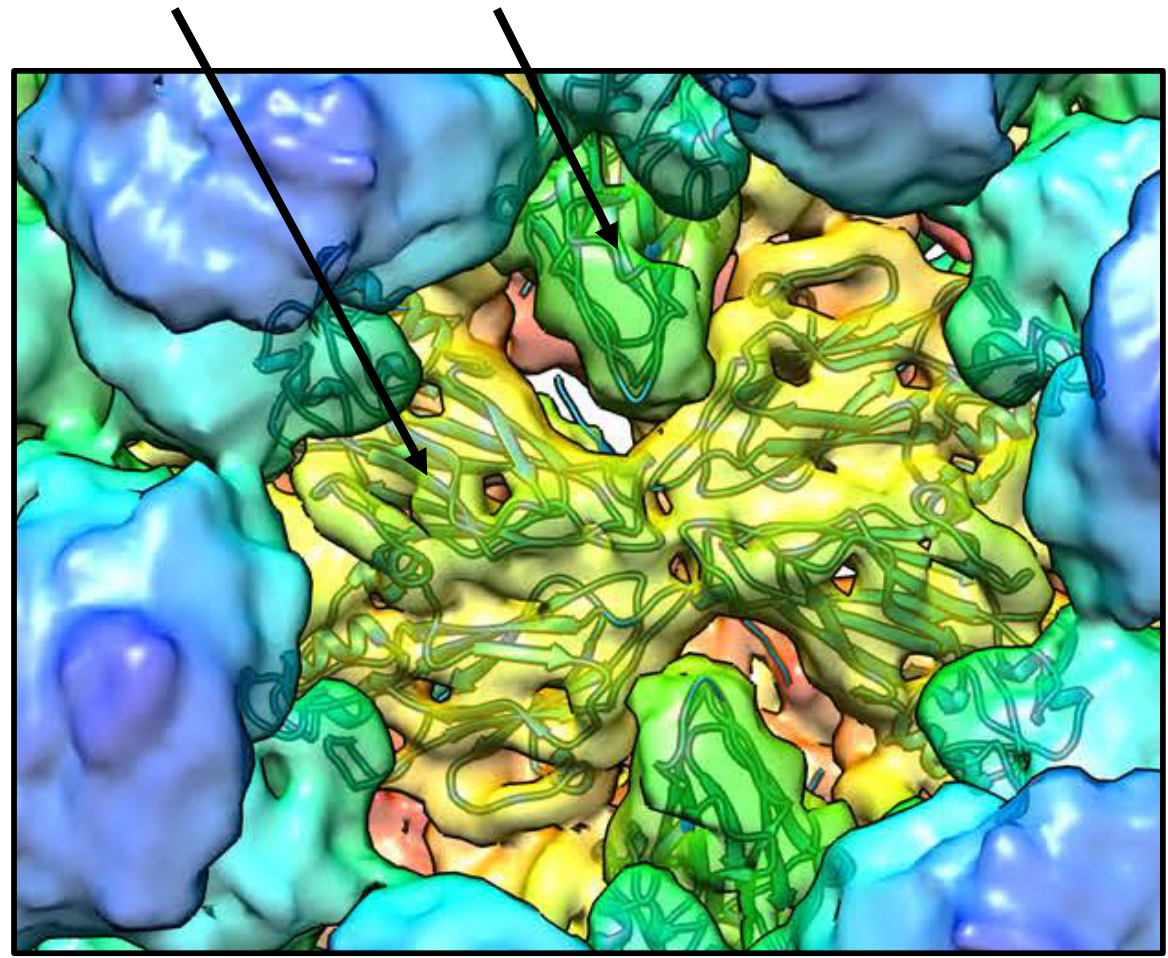




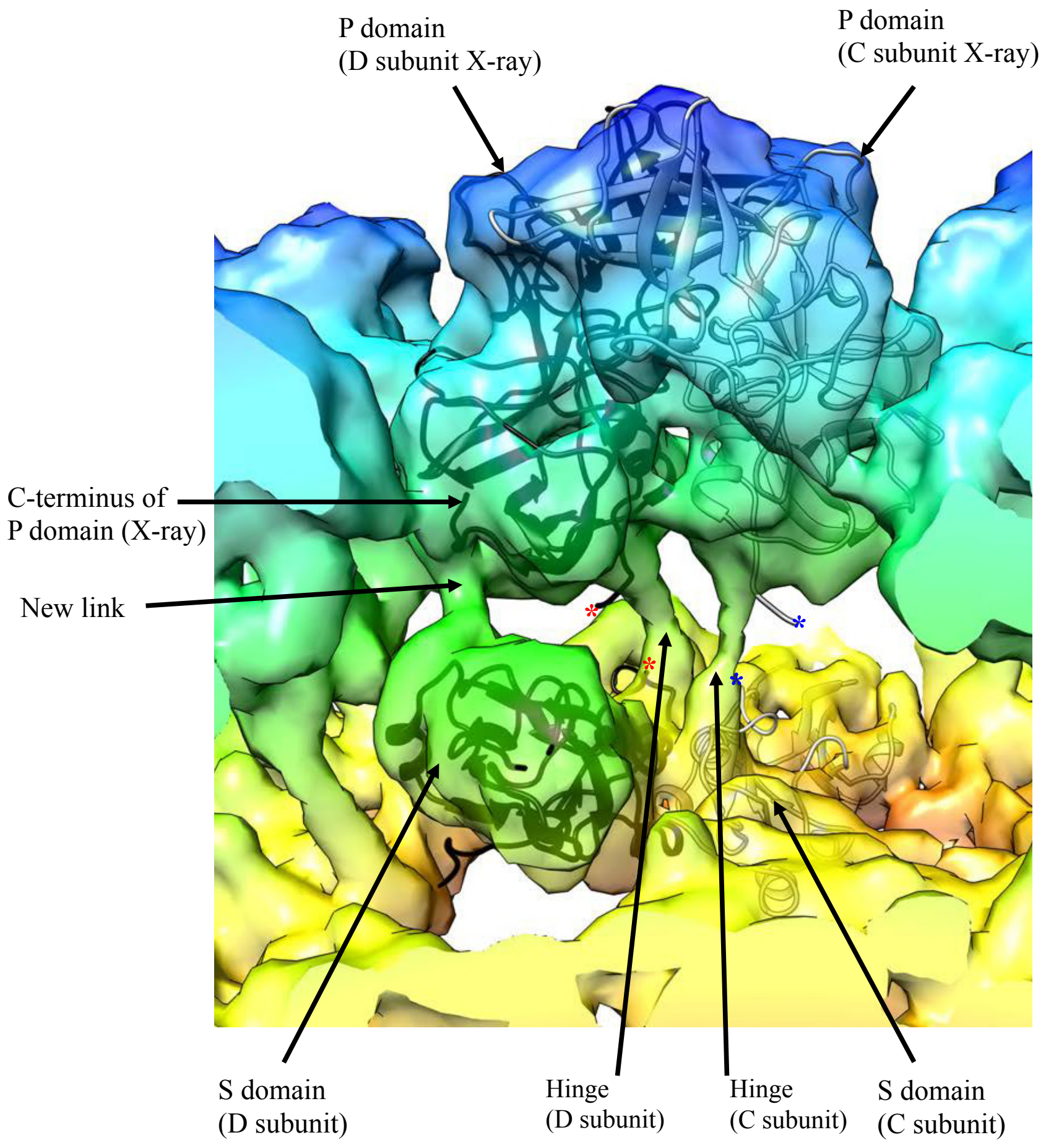


Figure 9

A

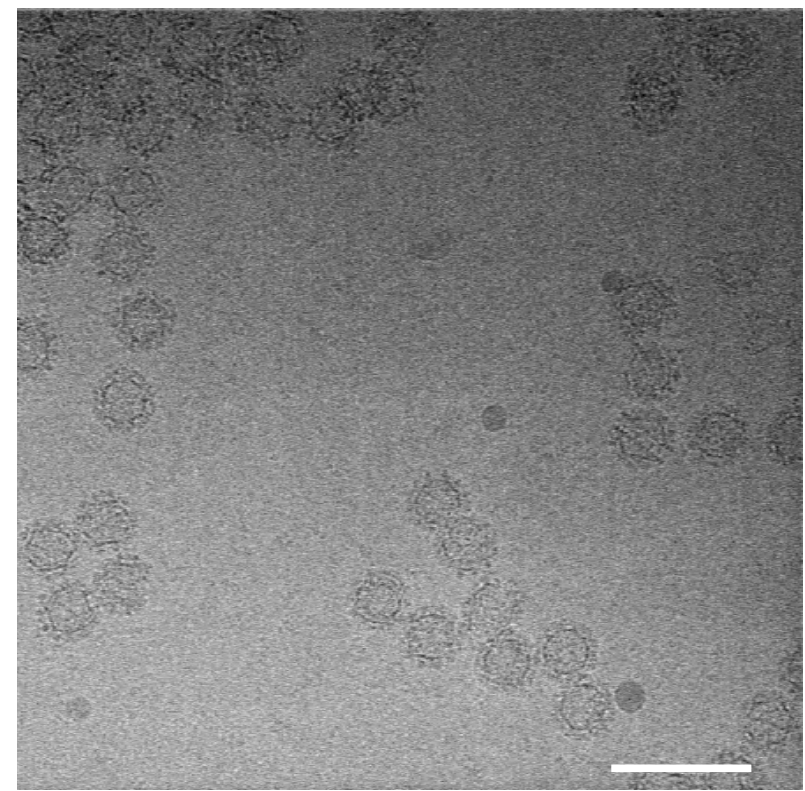

B

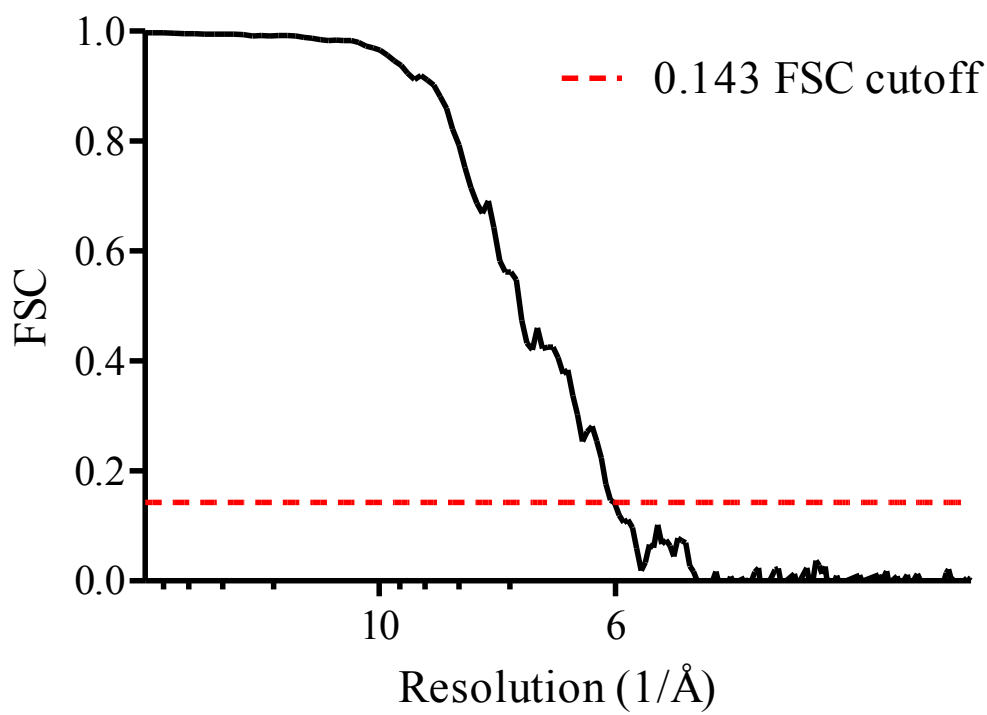


Figure 10
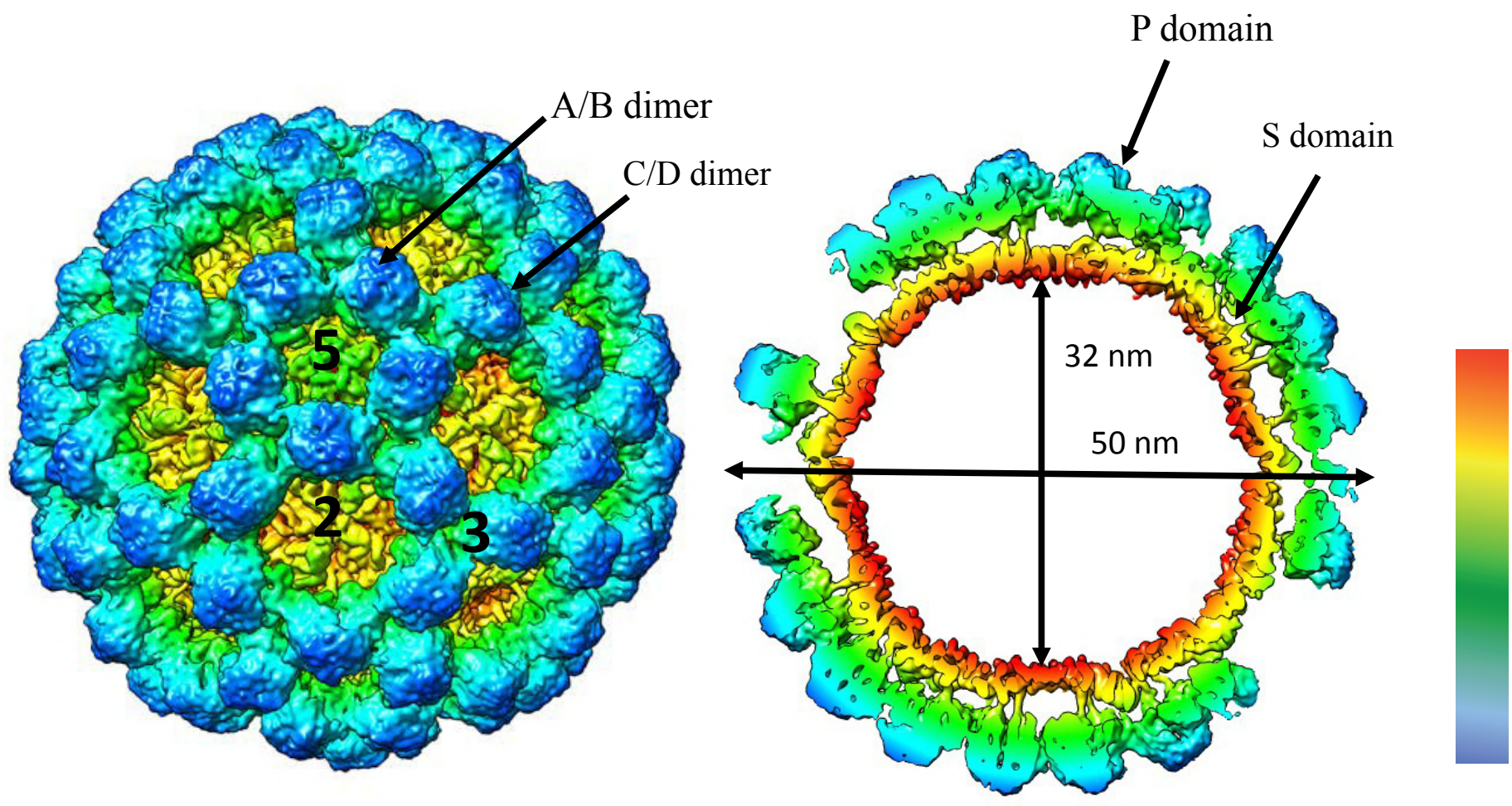

145- $\AA$

175-Å

200-Å

225-Å

254-Å 


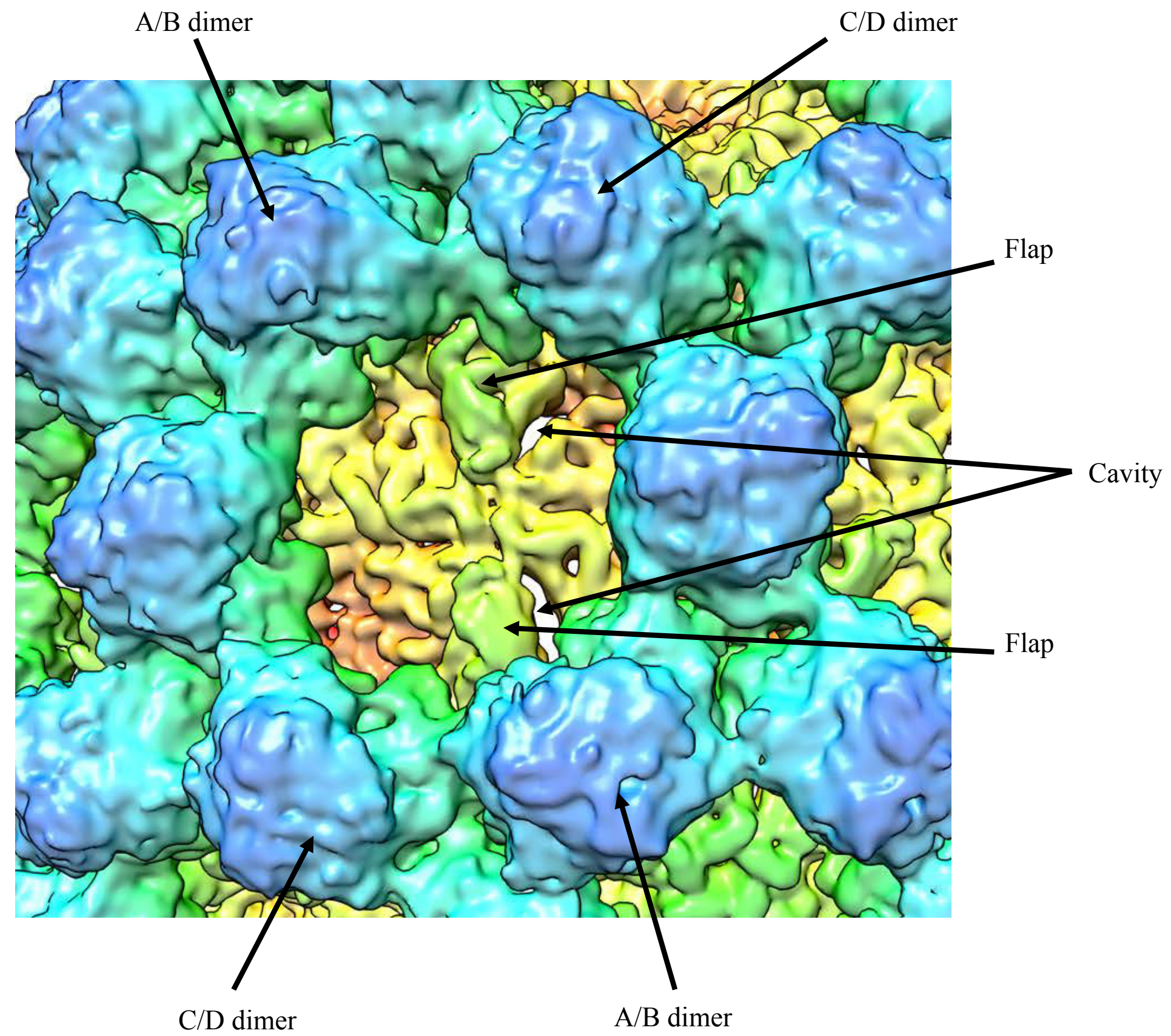


Figure 12

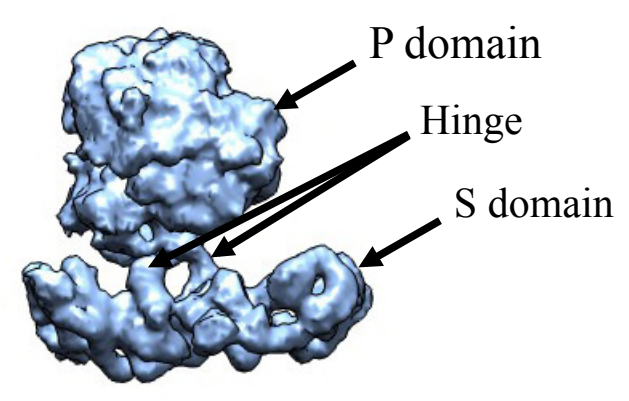

$\mathrm{A} / \mathrm{B}$ dimer

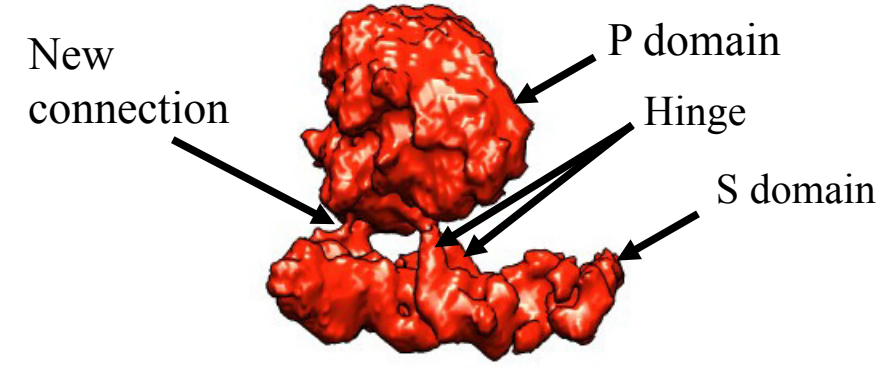

C/D dimer 
A
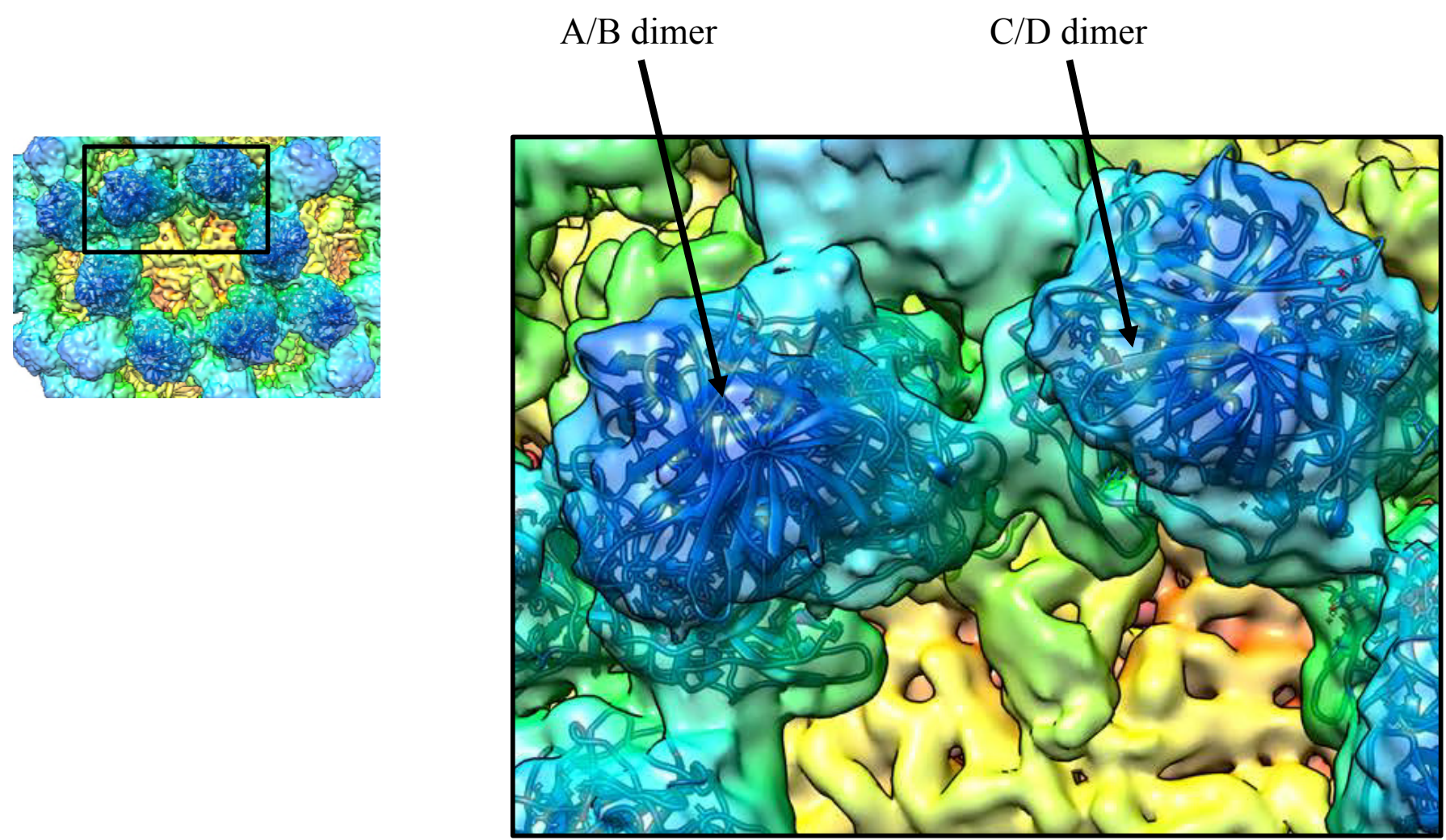

B
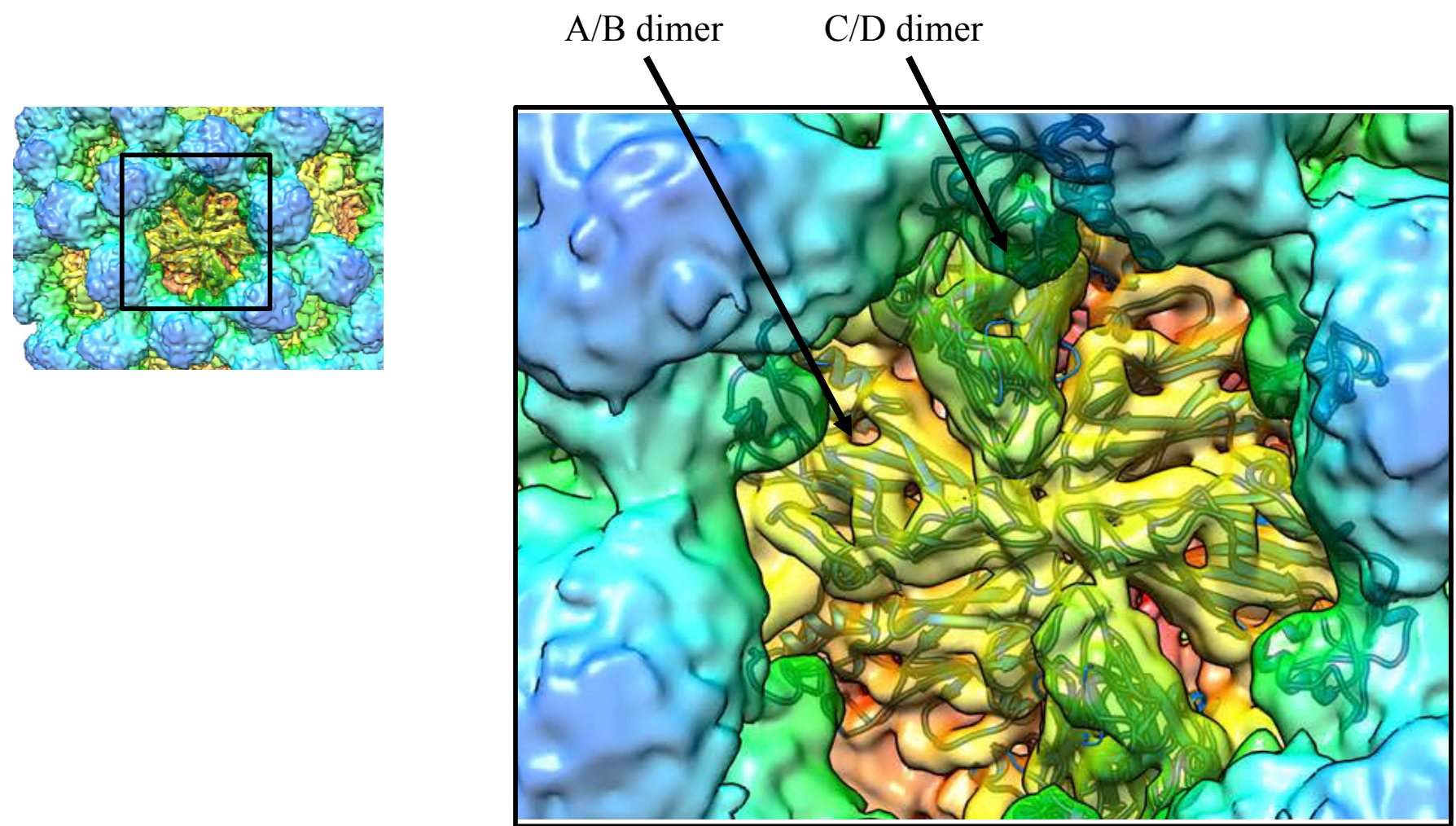


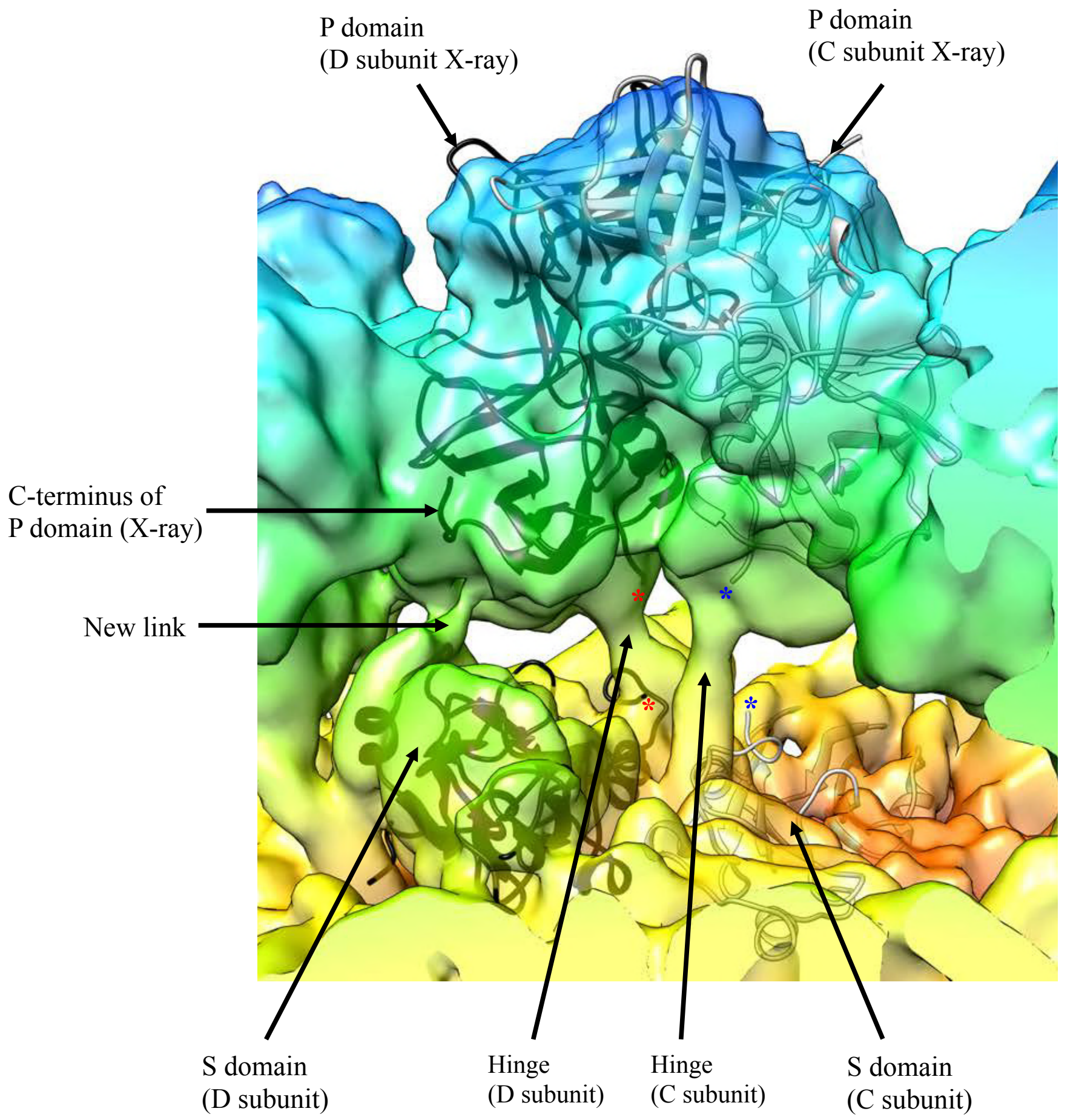


Figure 15
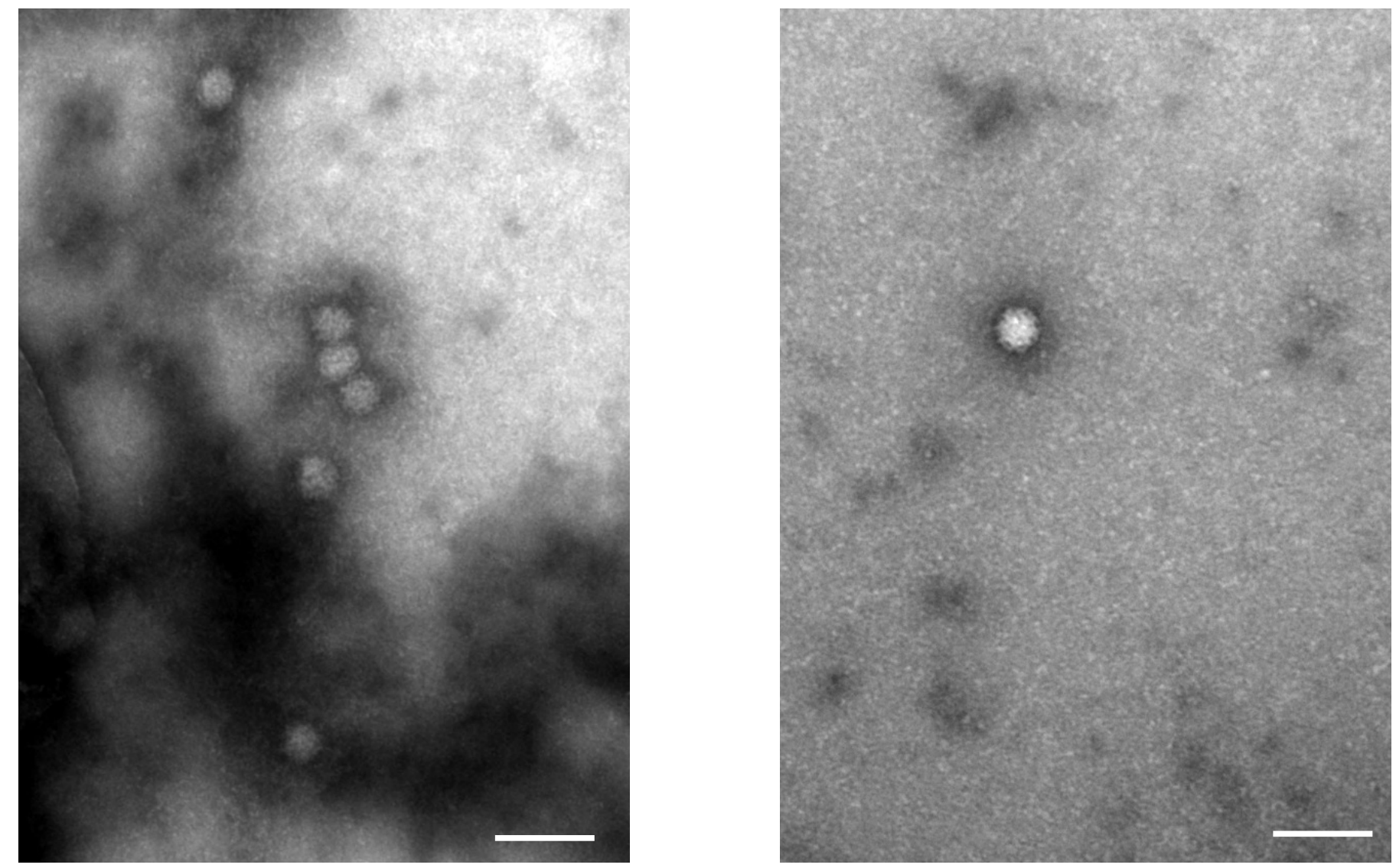